\title{
Generalization of Den Hartog's Equal-Peak Method for nonlinear primary systems
}

\author{
G. Habib ${ }^{a}$, T. Detroux, and G. Kerschen ${ }^{b}$ \\ Space Structures and System Laboratory \\ Department of Aerospace and Mechanical Engineering \\ University of Liège, Liège, Belgium.
}

\begin{abstract}
This study addresses the mitigation of one problem nonlinear resonance of a mechanical system. In view of the narrow bandwidth of the classical linear tuned vibration absorber, a new nonlinear absorber, termed the nonlinear tuned vibration absorber (NLTVA), is introduced in this paper. One unconventional aspect of the NLTVA is that the mathematical form of its restoring force is tailored according to the nonlinear restoring force of the primary system. The NLTVA parameters are then determined using a nonlinear generalization of Den Hartog's equal-peak method. The mitigation of the resonant vibrations of a Duffing oscillator is considered to illustrate the proposed developments.
\end{abstract}

\section{Introduction}

With continual interest in expanding the performance envelope of engineering systems, nonlinear components are increasingly utilized in real-world applications. Mitigating the resonant vibrations of nonlinear structures is therefore becoming a problem of great practical significance; it is the focus of the present study.

Nonlinear vibration absorbers, including the autoparametric vibration absorber [1], the nonlinear energy sink (NES) [2-4] and other variants [5-7], can absorb disturbances in wide ranges of frequencies due to their increased bandwidth. For instance, it was shown that an NES, i.e., an essentially nonlinear absorber, can extract energy from virtually any mode of a host structure [8]. The NES can also carry out targeted energy transfer, which is an irreversible channeling of vibrational energy from the host structure to the absorber [9]. This makes nonlinear vibration absorbers suitable candidates for vibration mitigation of nonlinear primary structures. However, the performance of existing nonlinear vibration absorbers is known to exhibit marked sensitivity to motion amplitudes. For instance, there exists a well-defined threshold of input energy below which no significant energy dissipation can be induced in an NES [3]. Likewise, the saturation phenomenon - characteristic of autoparametric vibration absorbers - occurs only when the forcing amplitude exceeds a certain threshold [1].

This paper builds upon previous developments [10,11] to introduce a new nonlinear vibration absorber for mitigating the vibrations around one problem nonlinear resonance. The absorber is termed the nonlinear tuned vibration absorber (NLTVA), because its nonlinear restoring force is determined according to the nonlinear restoring force of the host structure. In other words, we propose to

\footnotetext{
a e-mail: giuseppe.habib@ulg.ac.be

b The authors G. Habib, T. Detroux and G. Kerschen would like to acknowledge the financial support of the European Union (ERC Starting Grant NoVib-307265).
}

synthesize the absorber's load-deflection characteristic so that the NLTVA can mitigate the considered nonlinear resonance in wide ranges of motion amplitudes.

Furthermore, a nonlinear generalization of Den Hartog's equal-peak method for determining the NLTVA parameters is developed. The basic idea is to select the nonlinear coefficient of the absorber that ensures equal peaks in the nonlinear receptance function for an as large as possible range of forcing amplitudes. We will show that this is only feasible when the mathematical form of the NLTVA's restoring force is carefully chosen, which justifies the proposed synthesis of the absorber's load-deflection curve.

The paper is organized as follows. Sect. 2 briefly reviews Den Hartog's equal-peak method and revisits the dynamics of the classical linear tuned vibration absorber coupled to a Duffing oscillator. Sect. 3 lays down the foundations of the NLTVA by proposing a tuning rule for the absorber's restoring force. The NLTVA parameters are then determined using a nonlinear generalization of Den Hartog's equal-peak method. The conclusions of the present study are summarized in Sect. 4.

\section{The linear tuned vibration absorber (LTVA)}

The steady-state response of an undamped mass-spring system subjected to a harmonic excitation at a constant frequency can be suppressed using an undamped linear tuned vibration absorber (LTVA), as proposed by Frahm in 1909 [12]. However, the LTVA performance deteriorates significantly when the excitation frequency varies. To improve the performance robustness, damping was introduced in the absorber [13]; Den Hartog [14] and Brock [15] derived approximate analytic formulas for the absorber stiffness and damping in order to minimize the maximal response of the system at the resonant frequencies, which is obtained making the two resonant peaks have the same amplitude. 


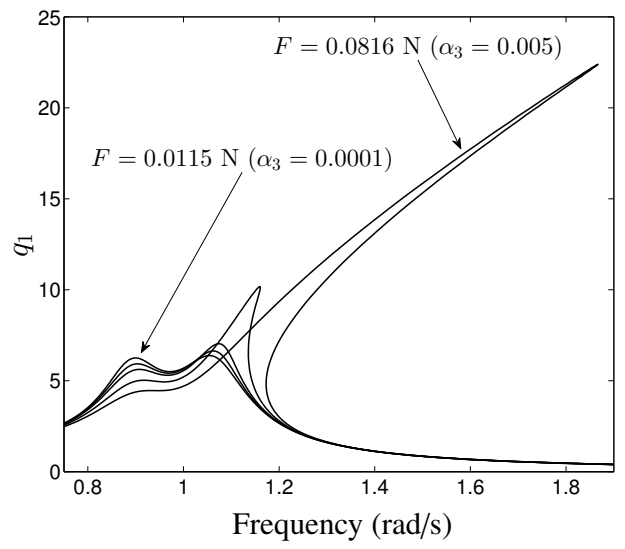

Fig. 1. Frequency response of a Duffing oscillator with an attached LTVA. $q_{1}$ is the dimensionless amplitude $\left(q_{1}=x_{1} k_{1} / F\right)$. For the computation $m_{1}=1 \mathrm{~kg}, c_{1}=0.002 \mathrm{~N} . \mathrm{s} / \mathrm{m}\left(\mu_{1}=0.001\right)$, $k_{1}=1 \mathrm{~N} / \mathrm{m}, k_{n l 1}=1 \mathrm{~N} / \mathrm{m}^{3}$ and $\epsilon=0.05$. For the different curves $F=0.0115 \mathrm{~N}, F=0.0258 \mathrm{~N}, F=0.0365 \mathrm{~N}, F=0.0577 \mathrm{~N}$, and $F=0.0816 \mathrm{~N}\left(\alpha_{3}=0.0001, \alpha_{3}=0.0005, \alpha_{3}=0.001\right.$, $\alpha_{3}=0.0025$ and $\alpha_{3}=0.005$, see later), where $F$ is the forcing amplitude.

Interestingly, it is only recently that an exact closed-form solution to this classic problem could be found [16]:

$$
\begin{aligned}
\lambda & =\frac{\omega_{n 2}}{\omega_{n 1}}=\sqrt{\frac{k_{2} m_{1}}{k_{1} m_{2}}}= \\
& =\frac{2}{1+\epsilon} \sqrt{\frac{2\left[16+23 \epsilon+9 \epsilon^{2}+2(2+\epsilon) \sqrt{4+3 \epsilon}\right]}{3\left(64+80 \epsilon+27 \epsilon^{2}\right)}} \\
\mu_{2} & =\frac{c_{2}}{2 \sqrt{k_{2} m_{2}}}=\frac{1}{4} \sqrt{\frac{8+9 \epsilon-4 \sqrt{4+3 \epsilon}}{1+\epsilon}}
\end{aligned}
$$

where $\omega_{n 1}$ and $\omega_{n 2}$ are the natural frequencies of the primary system and of the absorber, respectively, $\epsilon=m_{2} / m_{1}$ is the mass ratio, $\mu_{2}$ and $c_{2}$ are the damping ratio and damping coefficient and $k_{1}$ and $k_{2}$ are the spring coefficients of the primary system and of the absorber, respectively. This tuning condition minimizes the maximum response amplitude of the primary system.

The primary system considered throughout this paper is a harmonically-forced, lightly-damped Duffing oscillator. Fig. 1 shows the displacement response of the primary mass with an attached LTVA for various forcing amplitudes $F$, whose equations of motion are

$$
\begin{aligned}
m_{1} \ddot{x}_{1}+c_{1} \dot{x}_{1}+k_{1} x_{1}+k_{n l 1} x_{1}^{3} & +c_{2}\left(\dot{x}_{1}-\dot{x}_{2}\right) \\
& +k_{2}\left(x_{1}-x_{2}\right)=F \cos \omega t \\
m_{2} \ddot{x}_{2}+c_{2}\left(\dot{x}_{2}-\dot{x}_{1}\right)+ & k_{2}\left(x_{2}-x_{1}\right)=0 .
\end{aligned}
$$

The frequency response curves were computed using a pathfollowing algorithm combining shooting and pseudo-arclength continuation. The algorithm is similar to that used in [17]. In the figure it is visible that for low values of forcing amplitude $F$, the two resonant peaks have similar amplitude. However, increasing the forcing amplitude and practically activating the nonlinearity of the system, there is a clear detuning of the LTVA, i.e. one resonant peak largely increases its amplitude making the absorber ineffective.

\section{The nonlinear tuned vibration absorber (NLTVA)}

In view of the results presented in the previous section, it is meaningful to examine the performance of nonlinear absorbers for vibration mitigation of nonlinear primary structures. To mitigate a problem nonlinear resonance in an as large as possible range of forcing amplitudes, we introduce the nonlinear tuned vibration absorber (NLTVA). One unconventional feature of this absorber is that the mathematical form of its nonlinear restoring force is not imposed a priori, as it is the case for most existing nonlinear absorbers. Instead, we propose to fully exploit the additional design parameter offered by nonlinear devices and, hence, to synthesize the absorber's load-deflection curve according to the nonlinear restoring force of the primary structure.

\subsection{Synthesis of the nonlinear restoring force of the absorber}

The dynamics of a Duffing oscillator with an attached NLTVA is considered:

$$
\begin{aligned}
m_{1} \ddot{x}_{1}+c_{1} \dot{x}_{1}+k_{1} x_{1}+k_{n l 1} x_{1}^{3}+c_{2}\left(\dot{x}_{1}-\dot{x}_{2}\right) & \\
+g\left(x_{1}-x_{2}\right) & =F \cos \omega t \\
m_{2} \ddot{x}_{2}+c_{2}\left(\dot{x}_{2}-\dot{x}_{1}\right)-g\left(x_{1}-x_{2}\right) & =0
\end{aligned}
$$

where $x_{1}(t)$ and $x_{2}(t)$ are the displacements of the primary system and of the NLTVA, respectively. The NLTVA is assumed to have a generic smooth restoring force $g\left(x_{1}-x_{2}\right)$ with $g(0)=0$. We first define the dimensionless time $\tau=$ $\omega_{n 1} t$, where $\omega_{n 1}=\sqrt{k_{1} / m_{1}}$, and we apply the transformation $r(t)=x_{1}(t)-x_{2}(t)$, then, expanding $g(r)$ in Taylor series around $r=0$ and normalizing the system using $q_{1}=x_{1} / f$ and $q_{2}=r / f\left(f=F / k_{1}\right)$, we obtain

$$
\begin{aligned}
q_{1}^{\prime \prime}+2 \mu_{1} q_{1}^{\prime} & q_{1}+\frac{4}{3} \tilde{\alpha}_{3} f^{2} q_{1}^{3}+2 \mu_{2} \lambda \epsilon q_{2}^{\prime}+\lambda^{2} \epsilon q_{2} \\
& +\left.\frac{\epsilon}{m_{2} \omega_{n 1}^{2}} \sum_{k=2}^{\infty} \frac{f^{k-1}}{k !} \frac{\mathrm{d}^{k} g}{\mathrm{~d} r^{k}}\right|_{r=0} q_{2}^{k}=\cos \gamma \tau \\
q_{2}^{\prime \prime}+2 \mu_{1} q_{1}^{\prime} & +q_{1}+\frac{4}{3} \tilde{\alpha}_{3} f^{2} q_{1}^{3}+2 \mu_{2} \lambda(\epsilon+1) q_{2}^{\prime} \\
+\lambda^{2}(\epsilon+1) q_{2} & +\left.\frac{\epsilon+1}{m_{2} \omega_{n 1}^{2}} \sum_{k=2}^{\infty} \frac{f^{k-1}}{k !} \frac{\mathrm{d}^{k} g}{\mathrm{~d} r^{k}}\right|_{r=0} q_{2}^{k}=\cos \gamma \tau
\end{aligned}
$$

where prime denotes differentiation with respect to $\tau, 2 \mu_{1}=$ $c_{1} /\left(m_{1} \omega_{n 1}\right), \tilde{\alpha}_{3}=3 / 4 k_{n l 1} / k_{1}, 2 \mu_{2}=c_{2} /\left(m_{2} \omega_{n 2}\right), \epsilon=m_{2} / m_{1}$, $\lambda=\omega_{n 2} / \omega_{n 1}, \gamma=\omega / \omega_{n 1}$ and $\omega_{n 2}=\sqrt{\mathrm{d} g /\left.\mathrm{d} q_{2}\right|_{q_{2}=0} / m_{2}}$.

In Eqs. (4), the linear terms are independent of the forcing amplitude $f$, which confirms that a purely linear absorber attached to a linear oscillator is effective irrespective of the considered forcing amplitude. Focusing now on the complete system, $f$ appears in the nonlinear coefficients of both the primary system and the absorber, which reminds that it is equivalent to consider the system strongly nonlinear or strongly excited. Specifically, Eqs. (4) show that the forcing amplitude modifies linearly the quadratic terms, quadratically the cubic terms and so on. This suggests that, if an optimal set of absorber parameters is chosen for a specific value of $f$, variations of $f$ will detune the 
nonlinear absorber, unless the nonlinear coefficients of the primary system and of the absorber undergo a similar variation with $f$. According to Eqs. (4), this can be achieved by selecting the same mathematical function for the absorber as that of the primary system. When coupled to a Duffing oscillator, the NLTVA should therefore possess a cubic spring:

$$
\begin{gathered}
q_{1}^{\prime \prime}+2 \mu_{1} q_{1}^{\prime}+q_{1}+\frac{4}{3} \alpha_{3} q_{1}^{3}+2 \mu_{2} \lambda \epsilon q_{2}^{\prime}+\lambda^{2} \epsilon q_{2} \\
+\frac{4}{3} \epsilon \beta_{3} q_{2}^{3}=\cos \gamma \tau \\
q_{2}^{\prime \prime}+2 \mu_{1} q_{1}^{\prime}+q_{1}+\frac{4}{3} \alpha_{3} q_{1}^{3}+2 \mu_{2} \lambda(\epsilon+1) q_{2}^{\prime} \\
+\lambda^{2}(\epsilon+1) q_{2}+\frac{4}{3}(\epsilon+1) \beta_{3} q_{2}^{3}=\cos \gamma \tau
\end{gathered}
$$

where $\alpha_{3}=\tilde{\alpha}_{3} f^{2}$ and $\beta_{3}=\left.3 f^{2} g^{\prime \prime \prime}(r)\right|_{r=0} /\left(3 ! m_{2} \omega_{n 1}^{2} 4\right)$. The NLTVA should also possess a linear spring so that it is effective at low forcing amplitudes where the cubic spring of the Duffing oscillator is not activated.

In summary, the proposed nonlinear tuning rule is to choose the mathematical form of the NLTVA's restoring force so that it is a 'mirror' of the primary system.

\subsection{Nonlinear generalization of the equal-peak method}

The next objective is to determine the NLTVA parameters, namely $\epsilon, \lambda, \mu_{2}$ and $\beta_{3}$. The mass ratio $\epsilon$ is chosen according to practical constraints. The linear parameters $\lambda$ and $\mu_{2}$ are determined using Eqs. (1). Because an exact analytic estimation of $\beta_{3}$ is not within reach, an approximate solution is sought using the classic harmonic balance method limited to one harmonic component. The system is solved for fixed values of $\mu_{1}=0.001, \mu_{2}=0.134, \lambda=0.952$, for different values of $\epsilon$ and $\alpha_{3}$, and for a range of excitation frequencies $\gamma$ encompassing the system's resonances. Starting with weakly nonlinear regimes, i.e., $\alpha_{3}>0$, we seek the value of $\beta_{3}$, which gives two resonance peaks of equal amplitude. The procedure is repeated for increasing values of $\alpha_{3}$, which allows to consider stronger and stronger nonlinear regimes of motion.

The outcome of this numerical procedure is displayed in Fig. 2(a). This plot is interesting, because $\beta_{3}$ is almost linearly related to $\alpha_{3}$ for the different mass ratios considered, i.e., $\beta_{3} \cong a \alpha_{3}$. This linear relation implies that the nonlinear coefficient of the NLTVA that realizes equal peaks does not depend on forcing amplitude:

$$
\left.\beta_{3} \cong a \alpha_{3} \rightarrow \frac{3}{4} \frac{\left.f^{2} g^{\prime \prime \prime}(r)\right|_{r=0}}{3 ! m_{2} \omega_{n 1}^{2}} \cong a \frac{3}{4} \frac{f^{2} k_{n l 1}}{k_{1}} \rightarrow g^{\prime \prime \prime}(r)\right|_{r=0} \cong 6 a \epsilon k_{n l 1}
$$

The coefficient $a$ is determined by representing $\beta_{3}$ in function of $\epsilon$ for different values of $\alpha_{3}$, (Fig. 2(b)). It turns out that the regression $\beta_{3}=2 \alpha_{3} \epsilon /(1+4 \epsilon)$ provides an excellent approximation to the numerical results; so $a=2 \epsilon /(1+4 \epsilon)$.

Equations (5) are now solved considering this analytic expression of $\beta_{3}$ for different values of $\alpha_{3}$ and $\gamma$. We stress that the results presented in Fig. 2(c) were not computed using the one-term harmonic balance approximation but rather using the previously path-following algorithm mentioned in Sect. 2. This algorithm provides a very accurate numerical solution to the equations of motion. Fig. 2(c) shows that the NLTVA can enforce equal peaks in the frequency response $q_{1}$ of the Duffing oscillator for values of $\alpha_{3}$ ranging from 0.0001 to 0.005 . This result is remarkable in view of the variation of the resonance frequencies. For instance, the first resonance peak occurs at $\gamma=0.9$ for $\alpha_{3}=0.0001$ and beyond $\gamma=1$ for $\alpha_{3}=0.0075$. Another interesting observation is that the amplitude of the resonance peaks does not change substantially when $\alpha_{3}$ increases, which means that the response of the coupled system is almost proportional to the forcing amplitude, as it would be the case for a linear system. Conversely, Fig. 1 illustrates that for the same parameter values the LTVA is strongly detuned. All these results confirm the efficacy of the proposed NLTVA design.

In summary, given $m_{1}, c_{1}, k_{1}$ and $k_{n l 1}$ for a Duffing oscillator and given a mass ratio $\epsilon$, the NLTVA parameters can be determined using the following analytic formulas:

$$
\begin{aligned}
m_{2} & =\epsilon m_{1} \\
k_{2} & =\frac{8 \epsilon k_{1}\left[16+23 \epsilon+9 \epsilon^{2}+2(2+\epsilon) \sqrt{4+3 \epsilon}\right]}{3(1+\epsilon)^{2}\left(64+80 \epsilon+27 \epsilon^{2}\right)} \\
c_{2} & =\sqrt{\frac{k_{2} m_{2}(8+9 \epsilon-4 \sqrt{4+3 \epsilon})}{4(1+\epsilon)}} \\
k_{n l 2} & =\frac{2 \epsilon^{2} k_{n l 1}}{(1+4 \epsilon)}
\end{aligned}
$$

These formulas form the basis of a new tuning rule for nonlinear absorbers that can be considered as a nonlinear generalization of Den Hartog's equal-peak method.

For a more global comparison between the two absorbers, Fig. 3 represents the amplitude of the resonance peaks of the Duffing oscillator as a function of $F$. If the LTVA gets rapidly detuned, the amplitude of the two resonance peaks for the NLTVA remains almost identical. In addition, the amplitude is almost linearly related to forcing amplitude, as if the system would obey the superposition principle. This result is unexpected in view of the strongly nonlinear regimes investigated. It therefore seems that adding a properly chosen nonlinearity to an already nonlinear system can somehow linearize the dynamics of the coupled system. On the contrary, the amplitude of the resonance peaks for the LTVA exhibits a marked nonlinear dependence with respect to forcing amplitude.

For higher values of $\alpha_{3}\left(\alpha_{3} \approx 0.025\right.$ for $\left.\epsilon=0.05\right)$, also the NLTVA undergoes a detuning, qualitatively similar to the one occurring to the LTVA. In spite of this, the performances of the NLTVA are better than those of the LTVA for any value of $\alpha_{3}$. A detailed analysis of the detuning phenomenon is out of the scope of this paper.

Another phenomenon, not investigated in detail in this paper, but experienced during the research, is the appearance of quasiperiodic motions for certain values of $\alpha_{3}$ and $\gamma$. However, the amplitude of these motions appear to be not much larger than the predicted periodic solutions, thus these motions are not detrimental with respect to the functionality of the absorber.

\section{Conclusion}

Our purpose in this study is the development of a new nonlinear absorber, the NLTVA, for mitigating the vibrations 

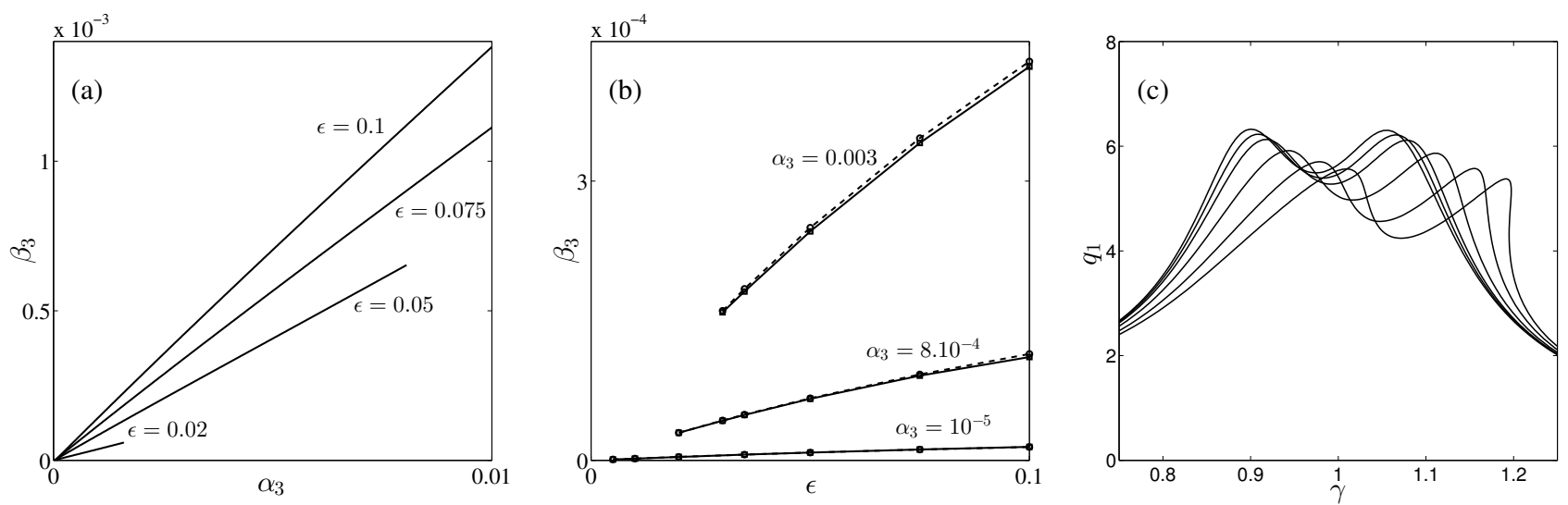

Fig. 2. Nonlinear equal-peak method. (a) Values of $\beta_{3}$ realizing equal peaks for increasing $\alpha_{3}$ and different $\epsilon$; (b) values of $\beta_{3}$ realizing equal peaks for increasing $\epsilon$ and different $\alpha_{3}$; the solid line is the result of the numerical computations, and the dashed line is the regression $\beta_{3}=2 \alpha_{3} \epsilon /\left(1+4 \epsilon\right.$ ). (c) Numerical solution of Eqs. (5) for $\epsilon=0.05, \mu_{1}=0.001, \mu_{2}=0.134, \lambda=0.952$. Curves from let to right: $\alpha_{3}=0.0001,0.0005,0.001,0.0025,0.005,0.0075$.

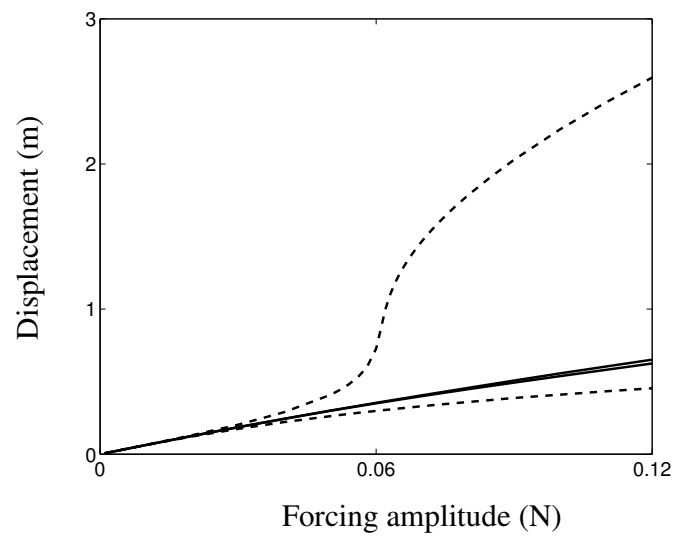

Fig. 3. Amplitude of the resonances peaks of the Duffing oscillator (solid lines: NLTVA, dashed lines: LTVA).

of a problem nonlinear resonance of a mechanical system. A specific objective is to ensure the effectiveness of the absorber in weakly as well as strongly nonlinear regimes of motion for which the primary system's resonance frequency can undergo substantial variations.

To this end, the additional design parameter offered by nonlinear devices, i.e., the mathematical form of the absorber's restoring force, is exploited thereby synthesizing nonlinearity for enhanced performance. We show that, if the NLTVA is 'a mirror' of the primary system, a nonlinear counterpart of Den Hartog's equal-peak method can be established. Simple analytic formulas, which are extremely accurate, are derived for this nonlinear equal-peak method. They lead to the design of an absorber with excellent performance in a relatively large range of forcing amplitudes. Interestingly, the coupled system Duffing-NLTVA exhibits dynamics that resemble to that of a linear system.

For very strongly nonlinear regimes, inherently nonlinear dynamical instabilities appear and detune the NLTVA. Despite these instabilities, the performance of the NLTVA remains always superior to the classical LTVA.

\section{References}

1. S. Oueini, A. Nayfeh, J. Vib. Contr. 6, (2000) 999-1016.
2. A.F. Vakakis, O.V. Gendelman, J. Appl. Mech. 68, (2001) 42-48.

3. A.F. Vakakis, O. Gendelman, L.A. Bergman, D.M. McFarland, G. Kerschen, Y.S. Lee, Nonlinear Targeted Energy Transfer in Mechanical and Structural Systems (Springer, 2009).

4. E. Gourdon, N.A. Alexander, C. Taylor, C.H. Lamarque, S. Pernot, J. Sound Vib. 300, (2007) 522-551.

5. J. Shaw, S.W. Shaw, A.G. Haddow, Int. J. Nonlin. Mech 24, (1989) 281-293.

6. S. Ema, E. Marui, Int. J. Mach. Tools Manuf. 36, (1996) 293-306.

7. N.A. Alexander, F. Schilder, J. Sound Vib. 319, (2009) 445-462.

8. G. Kerschen, J.J. Kowtko, D.M. McFarland, L.A. Bergman, A.F. Vakakis, Nonl. Dyn. 47, (2007) 285-309.

9. G. Kerschen, Y.S. Lee, A.F. Vakakis, D.M. McFarland, L.A. Bergman, SIAM J. App. Math. 66, (2006) 648-679.

10. R. Viguié, G. Kerschen, J. Sound Vib. 326, (2009) 780-793.

11. R. Viguié, G. Kerschen, J. Sound Vib. 329, (2010) 5225-5232.

12. H. Frahm, Device for Damping Vibrations of Bodies, US No. Patent 989958, 1909.

13. J. Ormondroyd, J.P. Den Hartog, Trans. ASME 50, (1928) 9-22.

14. J.P. Den Hartog, Mechanical Vibrations (McGrawHill, New York 1934).

15. J.E. Brock, J. Appl. Mech. 13, pp. A284, 1946.

16. T. Asami, O. Nishihara, J. Vib. Acoust. 125, (2003) 398-405.

17. M. Peeters, R. Viguié, G. Sérandour, G. Kerschen, J.C. Golinval, Mech. Sys. Sign. Proc. 23, (2009) 195-216.

18. G. Gatti, M.J. Brennan, I. Kovacic, Physica D 239, (2010) 591-599.

19. G. Gatti, M.J. Brennan, J. Sound Vib. 330, (2011) 4538-4550.

20. Y. Starosvetsky, O.V. Gendelman, J. Sound Vib. 315, (2008) 746-765.

21. T. Detroux, L. Renson, G. Kerschen, Proc. 32nd Int. Modal Analysis Conf., Orlando, USA, 2014. 